\title{
TETHERED FORMATION CONFIGURATIONS: MEETING THE SCIENTIFIC OBJECTIVES OF LARGE APERTURE AND INTERFEROMETRIC SCIENCE
}

\author{
Rodger E. Farley ${ }^{1}$ and David A. Quinn ${ }^{2}$
}

\begin{abstract}
With the success of the Hubble Space Telescope, it has become apparent that new frontiers of science and discovery are made every time an improvement in imaging resolution is made. For the HST working primarily in the visible and near-visible spectrum, this meant designing, building and launching a primary prirror approximately three meters in diameter. Conventional thinking tells us that accomplishing a mirror approximprovement in resolution at longer wavelengths for Earth and Space Science applications requires a corresponding increase in the size of the primary mirror. For wavelengths in the sub-millimeter range, a very large telescope with an effective aperture in excess of one kilometer in diameter would be needed to obtain high quality angular resolution. Realistically a single aperture this large is practically impossible. Fortunately such large apertures can be constructed synthetically. Possibly as few as three 34 meter formet longer wavelengths permitting not only very large virtual aperture science to be carried out, but high-resolution interferometry as well. To ensure the longest possible mission duration, a system of tethered spacecraft interferomely as well. To ensure the lon formation will will be needed to mitigate the need for a great deal of prope been proposed which possibly meet the likely meet these requirements. Sevi. This paper discusses two of them, weighing the relative pros and needs of the Space Science community. This pal being to settle on a configuration which combines the best features of structure, tethers and formation flying to meet the ambitious requirements necessary to make future large synthetic aperture and interferometric science missions successful.
\end{abstract}

\section{Introduction}

It follows intuition as well as optical theory that the larger the diameter of a telescope's mirror, the greater the amount of light that can be captured. That satisfies the quantity side of the equation, but as far as the quality side of the equation goes. a larger diameter mirror also implies a sharper image, which we would also intuitively expect. Here, quantity and quality have been used as euphemisms for sensitivity and resolving power. When faced with the desire for fine resolution and sufficient sensitivity to detect small dim objects (especially at long wavelengths), we find ourselves wanting impossibly large space observatories, and so we ask the question; is it possible to achieve both the quantitative and qualitative science objectives with realistically sized hardware? Fortunately, methods have been pioneered in the field of synthetic apertures, where small subapertures achieve resolutions comparable to a large mirror having a diameter equal to the spacing between the subapertures. The sensitivity is achieved by 'staring' at the target for a longer period of time, sampling different parts of the synthetic aperture. An exciting prospect is to merge the methods of a synthetic aperture with an imaging spectrometer optical system. The space science mission SPECS (Submillimeter Probe of the Evolution of Cosmic Structures: Mather et al. 2001) proposes to combine far-infrared interferometry in a synthetic aperture 1000 meters in diameter to produce a space platform imaging spectroscope. The heart of the instrument is a cryogenic Michelson interferometer with a stroking optical delay line. This type of optical set-up can detect spatial structures having time-invarient (during the observation), incoherent sources and has the ability to measure two basic properties:

1) Spatial brightness distribution

Aerospace Engineer, Mechanical Systems Center, Code 543, NASA Goddard Space Flight Center, Greenbelt, MD 20771

2 Aerospace Engineer, Guidance-Navigation and Control Center, Code 572, NASA Goddard Space Flight Center, Greenbelt, MD

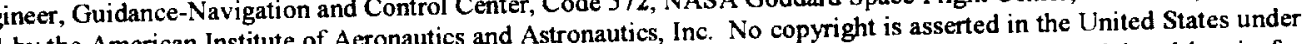
Copyright 2001 by the American Institute of Aeronautics and Astronautics, Inc. No copyright is asserte copyright claimed herein for Title 17, U.S. Code. The U.S. Government has a royalty-free license to exerci 
2) The spectrum in each resolution element (detector pixel)

The distance (baseline) between the subapertures makes the interferometer sensitive to a corresponding angular size of spatial structure. Interference fringe patterns (interferograms) are produced during the stroke cycle. which proceeds at a constant scan rate. The level of contrast (fringe visibility) between the light and dark fringes implies the amount of spatial structure (the brightness) at the angular size to which the interferometer is sensitive. Information contained in the shape of the fringe pattern corresponds to the spectrum of the object. The fringe shape is measured by varying the optical delay, and therefore during the stroke the intensity at a pixel location varies with time. This pattern is Fourier inverted to obtain the spectrum of the source. The synthetic aperture is sampled with three subapertures connected together using tethers to slowly spiral in like a record player. completely covering the plane of the aperture (the $u-v$ plane). The signals and subaperture locations in the synthetic aperture plane are Fourier transformed to create a sky map image with a spectrum of a particular brightness in each pixel. The image resolution is proportional to the synthetic aperture diameter, and the spectral resolution is proportional to the length of the stroking optical delay. The subaperture diameter of 3 meters limits the field of view, but SPECS will use a mosaic technique to produce wide-field images. The data needed to produce wide-field images are obtained when individual fringe patterns fade in and out over pixels in the detector array as the geometric delay corresponding to looking offaxis is compensated by the interferometer's optical delay. This occurs as the zero-path lengths of the sources are reached with the stroking of the delay line. The synthetic aperture is fully sampled by altering the baseline such that all angular sizes of interest are 'exposed.' In this way, all the components of the Fourier decomposition are measured which are used to produce the sky image.

Spinning tethered spacecraft formations hold the promise of making a SPECS observatory and others with a similar imaging need possible. The spin produces a centripetal acceleration, which keeps a tethered arrangement tight. This opens up tethered arrangements to the imagination, where tethered creations could resemble string-art-in-space, the 'artificial-gravity' of the spin maintaining tension and shape. Slow spin rates producing sub-Newton tension forces is all that is required to maintain shape. Precision to the level of interferometry is achieved by using optical path-length corrector mechanisms to make up for the small residual motions. Feedback for the corrector mechanism could come from a source in the field of view, where the 'white light' wave front maximum is detected.

There is a stark contrast between a spinning tethered formation and a free-flying formation in the area of fuel consumption. The tethered arrangements maintain a constant angular momentum, 'reeling' in or out their tethered lengths with winch-like mechanisms to fully sample the synthetic aperture by spiraling in and out. There is no use of fuel except to minimize perturbations. Only in repointing the formation by precession is fuel consumed. By contrast, a free-flying formation sampling in the same spiral manner for a SPECS mission would consume a fuel mass that was 3 times greater than the mass of the 'empty' spacecraft. just for one observation. The thrusters would have to perform the mechanical work equivalent to that done by tension in the tethers. Clearly, free flying formations for this kind of aperture sampling are completely impractical.

Spinning tethered arrangements can fit into two major categories: those in which the component spacecrafts are connected radially using a single tether (pendulum mode), or, those which have multiple attachments, allowing triangular or other 2-D geometrical shapes to define the arrangement. Complex arrangements can be fashioned with tension-compression structures that can be utilized to create 3-D shapes. But simplicity is a goal worth striving for, and the 2-D arrangements would have a much better chance of surviving intact after the building phase, the assembly phase, and the deployment phase, not to mention launch mass constraints. Key features of a 2-D spinning space tether arrangement are the over-all light mass of the system considering the immense size, using only centripetal force and strings, and it's open-loop dynamic stability. The beauty is the simplicity.

\section{Configurations}

Maintaining a constant angular momentum for the tethered system again minimizes the need for propulsion, but one problem with tethered arrangements having only subaperture masses, is the 'ballerina' effect. As the masses move radially closer to the center, the spin rate increases by one over the square of the radius, moving the subapertures faster than the sampling time required to minimize image blur. Thus, to tame the spin speed by not using propellant, one must use counter masses in the tethered arrangement. Such is the case for the two configurations studied. They each have 3 subapertures, and 3 counter masses. 


\section{SPECS Hex}

This arrangement is a pendulum type, with a central hub truss structure approximately 17 meters in radius. As the pendulum-connected subaperture reels out, the counter mass reels in with a 1:1 ratio. The radial reel-in speed required to sample the aperture plane in $\sim 10^{5}$ seconds, is $2.5 \mathrm{~mm} / \mathrm{s}$ with an initial rotational speed of 0.0165 RPM. This produces a Coriolis acceleration in which the reeled-in element will lead the hub attachment point by an angle, and the reeled-out element will lag by the same angle. That way. the subaperture and countermass tethered elements approach each other by twice the angle in the spin plane. The central hub radius was picked so as to keep the total approach angle (lead + lag) no greater than 20 degrees. The initial radial length of the tethers is $577 \mathrm{~m}$ from the hub center, which is a baseline of $1000 \mathrm{~m}$.

Figure 1 SPECS HEX

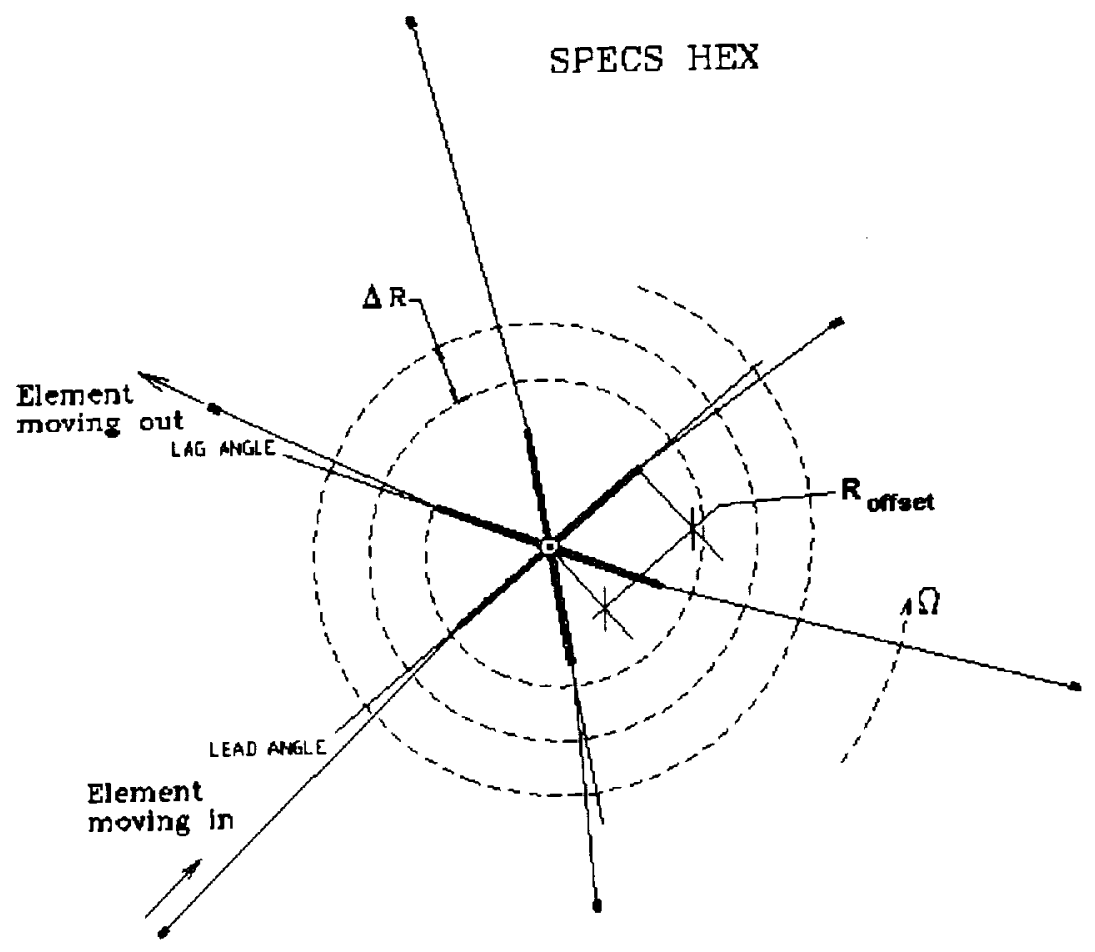

SPECS Tetra-Star

Use is made of triangles as the basic shape to give the tethered arrangement in-plane shape rigidity. The 3 counter masses are located at the apex of the outer 3 triangles, in which the two legs of each outer triangle are of a constant length tether. The base of the outer triangles form the inner triangle, where the subapertures are located in each corner. The length of this tether constitutes the interferometer baseline, and it is these three tethers which change length to spiral in and out, to fully sample the u-v plane. Each of the 3 inner tethers has a tether-winch on one end, and a passive in-line spring/damper suspension system on the other end. The triangular configuration allows passive damping to be used to great effect. For a standard observation, the reel-in rate of the inner tethers is $4.28 \mathrm{~mm} / \mathrm{s}$, with an initial rotational speed of 0.0165 RPM.

Figure 2

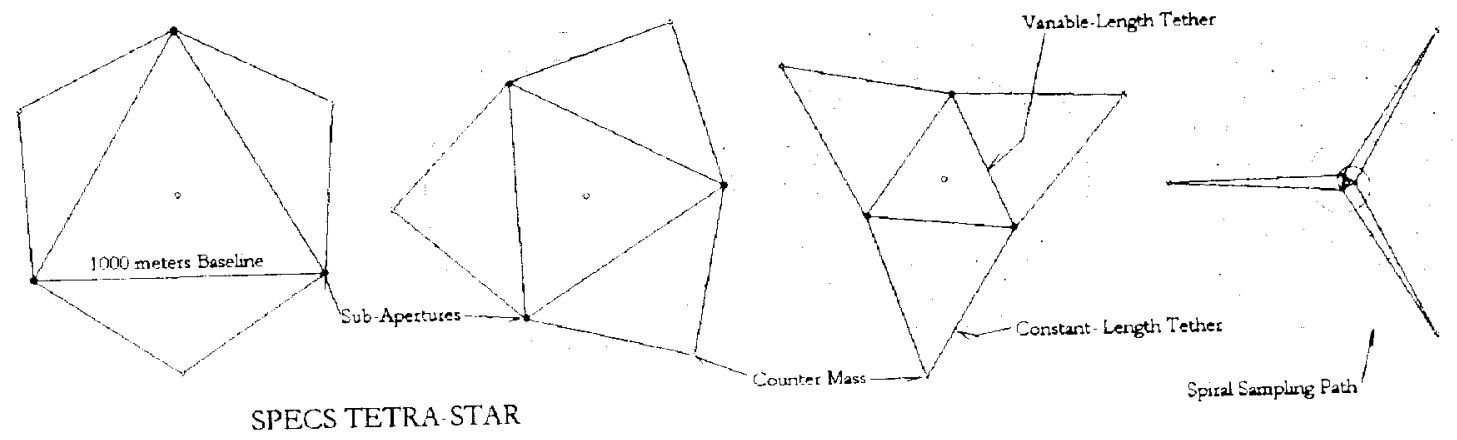




\section{Properties}

The tether material for the configurations was baselined as having a Young's modulus of 12 million psi. with an equivalent diameter of $1 \mathrm{~mm}$.

The mass of the subapertures, were optimistically selected to be $500 \mathrm{~kg}$ each. while the counter masses were set to $150 \mathrm{~kg}$. The actual SPECS subaperture is expected to be of much greater mass. It can be expected to have the following components:

1) A 3 or 4 meter diameter cryogenically cooled flat mirror.

2) Two 4 meter diameter thermally isolated / insulated stray-light baffle tubes.

3) Twelve 3-stage Adiabatic De-magnetization Refrigerators (ADR) connected to

4) Twelve cryo pump coolers. all heat sinked via cryo loop heat pipes to

5) A large thermal radiator.

6) Structure, tether mechanisms, perhaps an articulation mechanism for the mirror.

7) Sun shade with a solar array on the sunny side, the rest of the $s / c$ in the dark.

8) Electrical system, Thruster system, Attitude control system. Communication system.

\section{General Requirements for SPECS Tethered Configuration}

Spiral sampling will cover the synthetic aperture plane without gaps down to a minimum baseline. This can be accomplished by spiraling in, spiraling out, or a combination of the two maneuvers.

The sampling baseline (length from subaperture to subaperture) will vary from a minimum of 50 meters to a maximum of 1000 meters.

\section{Number of subapertures is 3 .}

The subapertures are folding-flats, having a 3 to 4 meter projected diameter.

To avoid image blur, maximum tangential velocity of the subapertures due to the spin is $1 \mathrm{~m} / \mathrm{s}$. For time efficient observations, this tip speed should be kept as close to $1 \mathrm{~m} / \mathrm{s}$ during the entire sampling sequence.

Angular stability of reflecting mirror relative to the other subaperture mirrors during an observation is 1 arc-second.

Temperature of the mirrors and any part of the system in the field of view $=<4 \mathrm{deg} \mathrm{K}$ (SPECS).
Tethered bodies should have a combined [radial plus out-of-plane] displacement $<0.5$ meters. At this range, the path length corrector mechanism can operate.

Knowledge of the position of the tethered subapertures: to a fraction of a wavelength, or about 10 micrometers in the case of SPECS.

The number of observations in a mission $=1500$

The average re-point change between observations $=5$ degrees

The total re-point angle for a mission $=7500$ $(5 \times 1500)$ degrees

\section{Dynamic Modeling}

The equations of motion were derived using Kane's method with the aid of AutoLev, a symbolic manipulation software designed specifically for solving dynamics problems. The SPECS Hex model has 24 degrees of freedom (dof), and the SPECS Tetra Star has 18 dof. Both models used point masses to represent the subapertures and counter-mass spacecraft, and the tethers were modeled as massless springs. Considering that the tension is never greater than several Newtons. the typical tether mass is perhaps on the order of $1 \%$ of the counter mass or subaperture mass, so a massless assumption does not lead one far astray. However, this does mean that the transverse tether vibrations will not be formulated. The transverse vibrations are estimated to have a base period of 10 seconds (guitar mode). where as the spin period is between 15 and 60 minutes. The equations were numerically integrated using a $5^{\text {th }}$ order Runge-Kutta with a constant time increment and animated in Visual Basic software. Even though simplifying assumptions were used, the computer code generated is extremely complex, especially the damping terms. AutoLev provides subroutines of the equations of motion in either Fortran or C. In the case of this study, the equations had to be manually altered to convert the Fortran code into Visual Basic code, with most of the effort in reformatting the line continuances.

\section{Maneuvers}

\section{Observation scan}

An observation scan entails spiraling in such a manner as to cover the $u-v$ plane. The $u-v$ plane is a

transformation from the physical aperture plane to one non-dimensionalized by the wavelength $\lambda$. If $x$ and $y$ are the physical coordinates in the aperture plane, then: $\mathrm{u}=\left(\mathrm{x}_{2}-\mathrm{x}_{1}\right) / \lambda, \mathrm{v}=\left(\mathrm{y}_{2}-\mathrm{y}_{1}\right) / \lambda$ 
where $x_{1}, y_{1}$ is the location of subaperture 1 , and $x_{2}, y_{2}$ is the location of subaperture 2 .

To physically cover the entire aperture, the spiral spacing $\Delta R$, should be a constant. See fig. 1 .

$\Delta R=D_{\text {sub }} \cdot N_{\text {sub }}$ where $D_{\text {sub }}$ is the diameter of the subaperture, and $\mathrm{N}_{\text {sub }}$ is the total number of subapertures.

Spiraling occurs as the tethers are reeled-in while the constellation is rotating at the instantaneous rate $\Omega$. The radial reel-in rate $\mathrm{dR} / \mathrm{dt}$ is designated as $\mathrm{Rdot}$. To make the spiral spacing a constant for efficient observations, it implies that the ratio $\mathrm{Rdot} / \Omega$ is a constant. Indeed:

$\mathrm{Rdot}=\Omega \cdot \Delta \mathrm{R} /(2 \pi)$. Rdot will vary during the observation, as $\Omega$ will vary with the radial position of the subapertures and counter-masses in this constant angular momentum system. The way to keep $\Delta \mathrm{R}$ a constant, is to vary Rdot by this relationship:

$\operatorname{Rdot}_{I}=\operatorname{Rdot}_{0} \cdot\left(I_{\text {spino }} / I_{\text {spinI }}\right)$, where Rdoto is the initial value of $R d o t$, and $I_{\text {spino }}$ is the initial value of system spin inertia. $\mathrm{I}_{\text {spin }}$ is the instantaneous system spin inertia, and $\mathrm{Rdot}_{1}$ is the instantaneous value of Rdot. If the tethered system spin inertia $I_{\text {spin }}$ is parameterized by $R$, the subaperture radial distance from the system center of mass. and $\mathrm{H}$ is the system angular momentum magnitude about the center of mass, then:

$R \operatorname{dot}(R)=H \cdot \Delta R /\left[2 \pi \cdot I_{\text {spin }}(R)\right]$

The minimum time (seconds) it takes to complete one fully sampled spiral observation, when the rule for keeping $\Delta \mathrm{R}$ a constant is practiced. can be calculated from this integral:

$$
T_{\text {observation }}=\frac{2 \cdot \pi}{H \cdot \Delta R} \cdot \int_{R_{\text {inner }}}^{R_{\text {outer }}} I_{\text {spin }}(R) d R
$$

Where $R_{\text {inner }}$ is the innermost radial distance that the subapertures will be reeled to, and $R_{\text {outer }}$ is the maximum radial position.

With the SPECS-hex configuration, the rigid hub structure requires a minimum structural radius $\mathbf{R}_{\text {offset }}$ to keep the Coriolis-induced angular deflections down to a required value during the observation. If the reel-in rate of the subapertures is equal to the reel-out rate of the counter-masses, then the Coriolis lead angle $\Psi_{\text {lead }}$ will equal the Coriolis lag angle $\Psi_{\text {lag. }}$. This means that a subaperture and counter-mass will approach each other by the angle $2 \Psi$ in the aperture plane. Therefore. if $\Psi_{\text {req }}$ is the maximum angle tolerable, then the required offset radius is:

$R_{\text {offset }}=\Delta R /\left(\pi \cdot \operatorname{Sin}\left(\Psi_{\text {req }}\right)\right)$

One can, in effect, determine the reel-in rate whereby this configuration will 'wrap itself around the axle'. If the lead and lag angles are 30 degrees, then all the tethers will converge together. For the baseline SPECS-hex configuration, that will happen when Rdot $=7.3 \mathrm{~mm} / \mathrm{s}$. This calculation demonstrates the extreme sensitivity of pendulum style tethered arrangements.

In-plane transient motion occurs with the stop-start action of the reel mechanism. This effect can be minimized with a ramp-up, ramp-down time, perhaps lasting several cycles of the axial vibration mode (1/3 to $1 \mathrm{~Hz}$ ). In-line dampers can attenuate the residual motions to an acceptable level.

\section{Re-pointing}

One method of repointing that has been suggested (but rejected) is to reel-in all the spinning masses, rigidly hook-up, then use thrusters to de-spin. After despinning, then re-point, re-spin, and re-deploy. This is too complex, and too costly in terms of fuel and time. An alternative that barely uses fuel is to precess the spinning disk like a top. It is quite possible to precess a spinning constellation of tethered objects in such a way that all the objects move as though they were one rigid object. The tethers need to be a small fraction of the total spin inertia, which in this case they are. To illustrate with a mental experiment, let us consider a rigid spinning disk with an axle in the center. We take a marker and draw a random grid pattern over the disk, symbolically dividing the disk into many individual elements of various sizes. We apply a constant lateral moment to the axle, and the spinning disk precesses at a constant rate. Now, what were the forces involved to keep all the elements moving in concert with one another, at the same rate? Each element must 'feel' a perturbing force perpendicular to the disk, proportional to the mass of the element $\mathrm{M}_{\mathrm{j}}$ in order to keep up with the other elements. Also, to keep up angularly, the force must be proportional to the radial distance from the center $R_{j}$. In addition, the applied moment on the spinning disk produces a harmonically varying force amplitude, with a period equal to the spin period. Each element has a phase angle $\phi_{j}$ corresponding to its relative angular position in the tethered arrangement.

Mathematically, the thrust required perpendicular to the spinning plane for each element $\mathrm{j}$, is thus: 
Thrust $\mathrm{j}_{\mathrm{j}}=\mathrm{M}_{\mathrm{j}} \cdot \mathrm{R}_{\mathrm{j}} \cdot \mathrm{C}_{\mathrm{T}} \cdot \sin \left(\Omega \cdot \mathrm{t}-\phi_{\mathrm{j}}\right)$, Newtons

Where $\Omega$ is the spin rate, radians/s and $\phi$ is the constant configuration phase angle for the element, radians. See Figure 3.

$\mathrm{C}_{\mathrm{T}}$ is a "specific thrust constant' with units of $\sec ^{-2}$

$\mathbf{C}_{\mathrm{T}}=\theta_{\mathrm{p}} \cdot \Omega^{2} /\left(\pi \cdot \mathrm{N}_{\mathrm{revs}}\right)$

Where $\theta_{\mathrm{p}}$ is the re-point angle to precess, radians. $\mathrm{N}_{\text {revs }}$ is the number of revolutions in which to complete the maneuver. This does not have to be a whole number.

The precession rate $\mathrm{d} \theta_{\mathrm{p}} / \mathrm{dt}=\mathrm{C}_{\mathrm{T}} / 2 \Omega$, radians $/ \mathrm{s}$

Figure 3

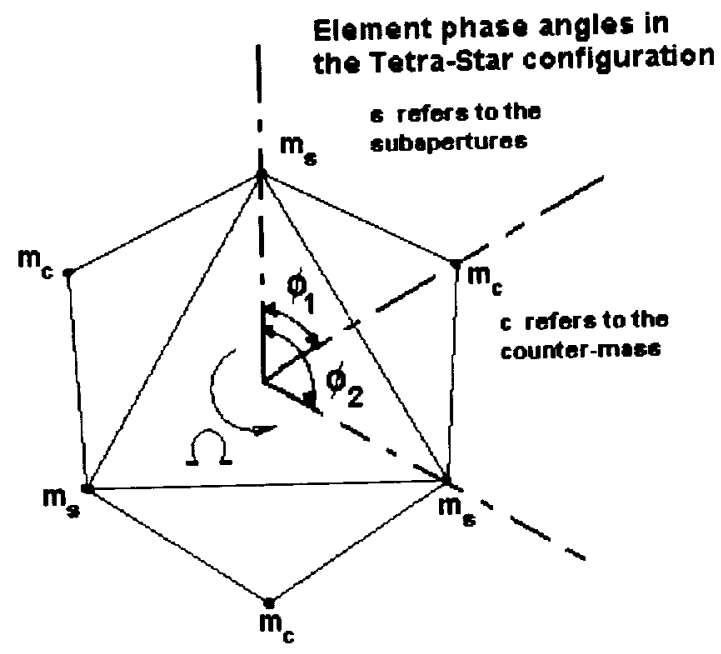

If the above set of precession rules are followed, then repointing can occur smoothly and quickly. The instantaneous spin plane normal direction is determined by averaging the vectors produced by this cross product:

$$
\overrightarrow{\text { Normal }}=\frac{\sum_{j=1}^{N}\left(\vec{R}_{j}-\vec{R}_{c m}\right) \times\left(\vec{v}_{j}-\vec{v}_{c m}\right)}{N}
$$

where $\mathrm{cm}$ refers to the system center of mass, and $\mathrm{N}$ is the total number of tethered elements. This is the instantaneous direction in which the thrusters are fired, the thrust magnitude varying harmonically.

\section{Fuel Usage}

The ratio of fuel mass to empty mass (spacecraft, less consumable fuel for repointing) equation has been derived for a tethered element, and has the following form: $\mathrm{m}_{\text {fuel }} / \mathrm{m}_{\mathrm{e}}=\left[\mathrm{e}^{(\mathrm{R} \cdot \Omega \cdot \theta \mathrm{p} /(\mathrm{g} \cdot \mathrm{Isp}))}-1\right]$ where Isp is the specific impulse for the fuel, and $g$ is the acceleration of gravity, $9.81 \mathrm{~m} / \mathrm{s}^{2}$. This is a simplified equation for pulse-thrusting the elements when they are at the correct location/phase. It underestimates the fuel for the real case of continuously varying thrust.

Another wonderful little equation that just slightly overestimates the fuel usage ( $4 \%$ over for the total mission, quite accurate for short duration burns) is:

$$
M_{\text {fuel }}=\frac{4}{\pi} \cdot \frac{\theta_{p} \cdot \Omega}{I s p \cdot g} \cdot\left(\sum_{j=1}^{N} M_{j} \cdot R_{j}\right)
$$

Where, the $\mathrm{Mj}$ and $\mathrm{Rj}$ are individual instantaneous element mass (fuel + empty) and radius in the configuration.

For a constant angular momentum system, a determination can be made as to the most efficient location for the elements in order to minimize the fuel usage per radian of re-point angle. For a single tethered element maintaining a constant angular momentum, the larger the radius $\mathrm{R}$, the less fuel is consumed to tilt the spinning plane a given angle. The ratio of fuel mass to empty mass equation for constant angular momentum has the following form:

$\mathrm{m}_{\text {fuel }} / \mathrm{m}_{\mathrm{e}}=\left[\mathrm{e}^{(\mathrm{k} \cdot \theta \mathrm{p} /(\mathrm{R} \cdot \mathrm{g} \cdot \mathrm{Isp}))}-1\right]$ where $\mathrm{k}=\Omega \mathrm{R}^{2}$. a constant of the initial condition. See Figure 4.

Using hydrazine as a fuel, Isp $=220$ seconds, and the total re-point angle $=7500$ degrees, for a single tethered element:

Figure 4 Single tether fuel usage to precess $7500^{\circ}$

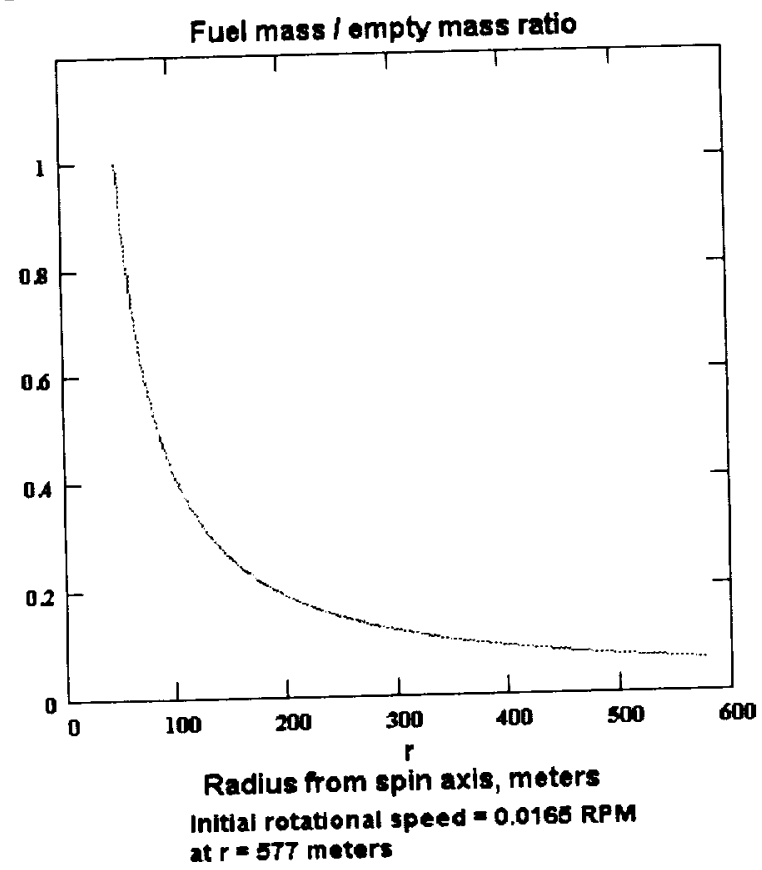


For the more complex case of a harmonically varying thrust, the just slightly over-estimating formula for fuel mass consumed is:

$$
M_{\text {fuel }}=\frac{4}{\pi} \cdot\left[\frac{\theta_{p} \cdot H}{\text { (Isp } \cdot g)}\right] \cdot \frac{\left(\sum_{j=1}^{N} M_{j} \cdot R_{j}\right)}{\left[\sum_{j=1}^{N} M_{j} \cdot\left(R_{j}\right)^{2}\right]}
$$

where $\mathrm{H}$ is the angular momentum of the tethered system.

For a configuration like the Tetra Star, the change of radial positions between the subapertures and countermasses progresses in a non-linear fashion, and a system determination of the fuel usage must be made. Figure 5 addresses this case.

Fuel mass consumed for 7500 degrees of precession for the Tetra-Star configuration, for various inner tether lengths at constant system angular momentum and $2100 \mathrm{~kg}$ initial system mass:

Figure 5 Tetra Star mission re-point fuel consumption.
The fuel mass to total mass fraction varies from $7.5 \%$ to $9.5 \%$ to accomplish the total mission-repointing requirement with the Tetra-Star configuration. If the baseline length during repointing is kept at 1000 meters, then $165 \mathrm{~kg}$ of fuel is consumed by the subapertures and counter masses combined, out of an initial system mass of $2100 \mathrm{~kg}$. The worst

configuration occurs at a baseline length of 330 meters, where $205 \mathrm{~kg}$ of fuel are required.

\section{Simulation Results}

The preliminary open-loop dynamic motion results for the Tetra-Star configuration are presented here. The simulations are separated into 2 categories: the observation mode, and the re-point mode. During the observation mode, the reel-in simulations determined the tension, tangential speed, coverage of the synthetic aperture plane (and thus u-v plane coverage), in-plane transient motions, and the total elapsed time for an observation. The initial conditions for the simulations did not include tether pre-stretch due to centrifugal force (in real life they would). This created an unrealistic initial transient 'breathing mode' in the tethered arrangement. However, this tested and illustrated the effectiveness of the passive damping shock struts at the tether's terminal attachment point. To spiral from a baseline of 1000 meters to 50 meters took 34.3 hours for an observation, exactly as calculated from the formula developed.

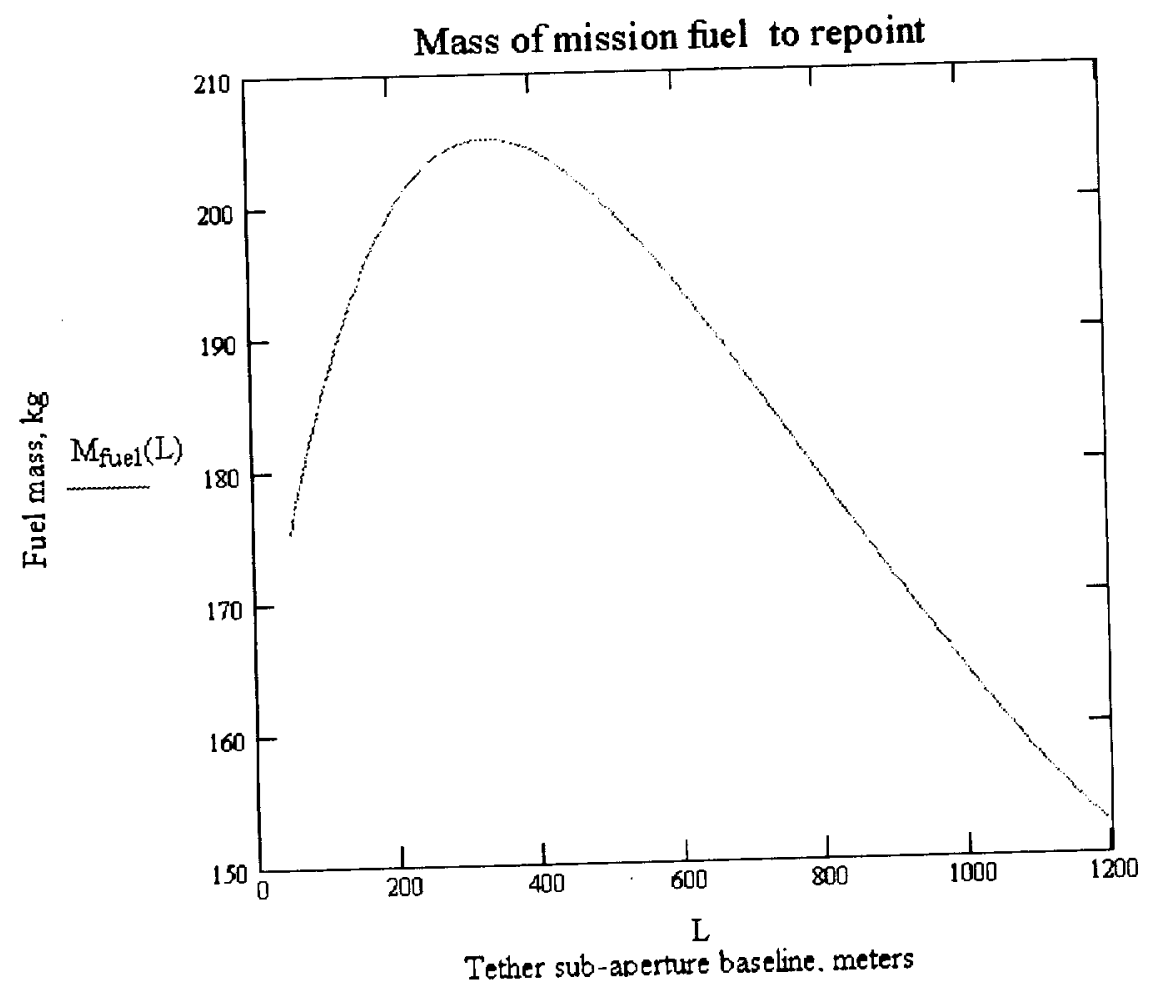


Observation Mode Results

Figure 6: Sub-aperture tangential speed as a function of time.

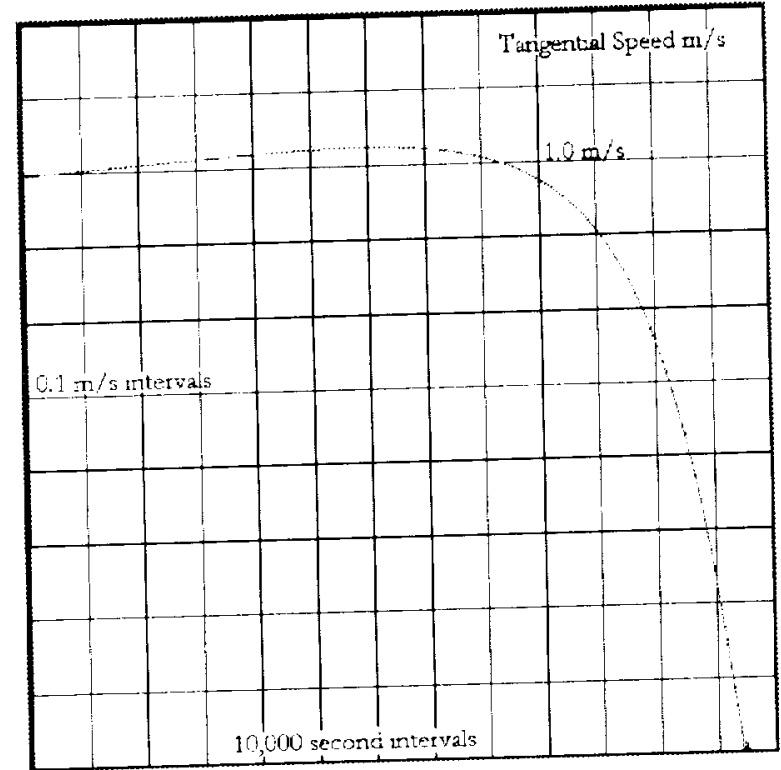

Figure 7: Sub-aperture tether tension as a function of time. Tether material stress is less than $200 \mathrm{psi}$.

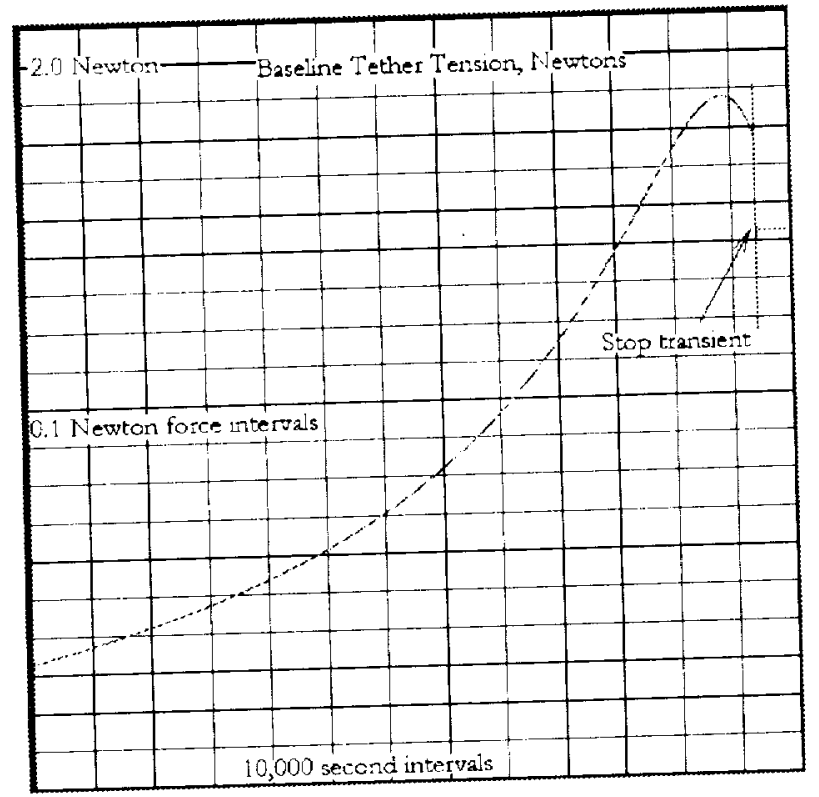

Figure 8: Sub-aperture tether stretch transient.

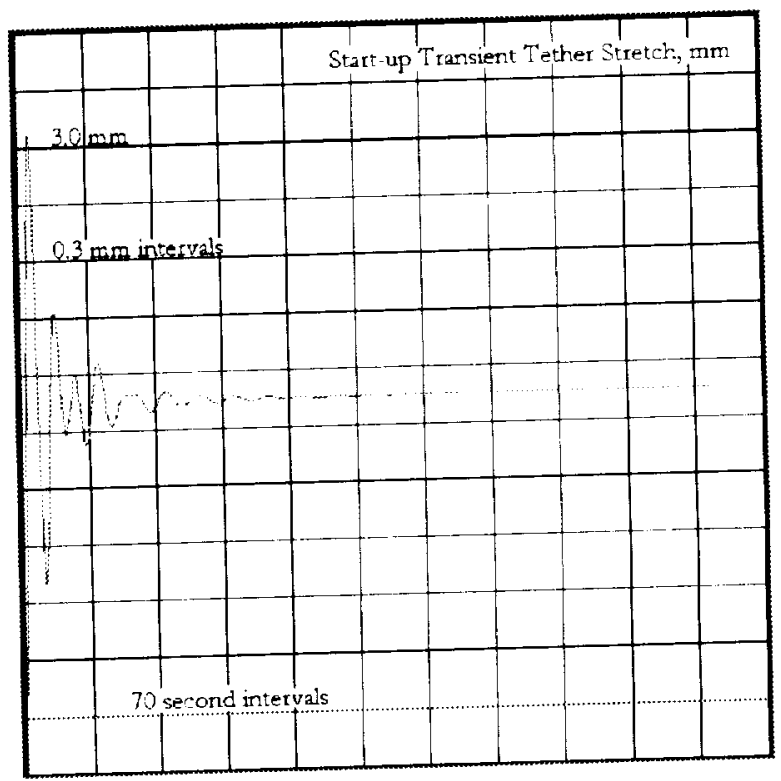

Figure 9: Sub-aperture tether stretch as a function of time.

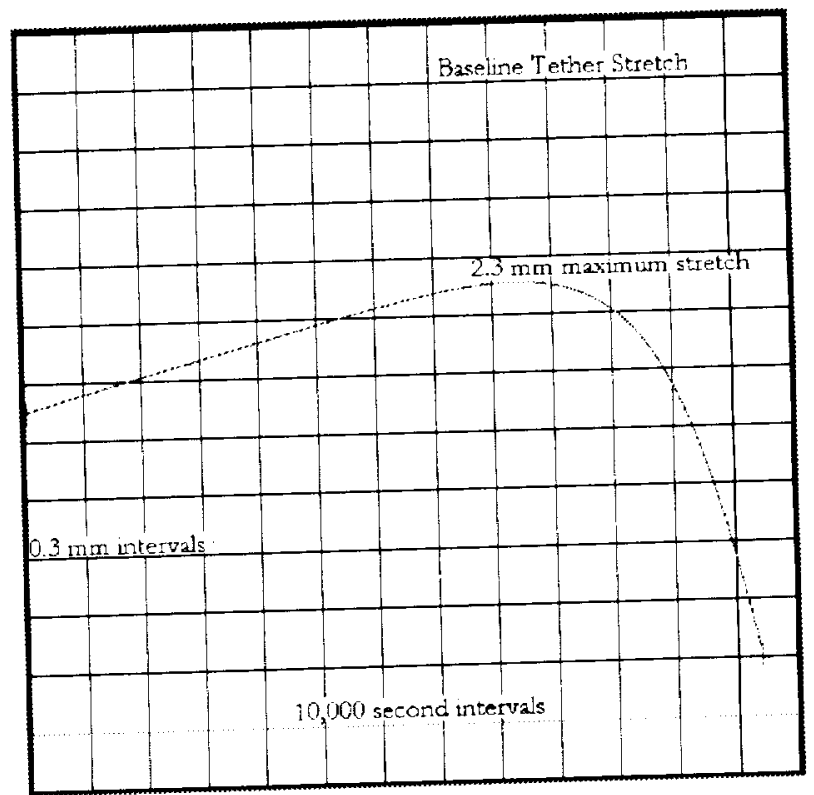




\section{Repointing Mode Results}

The simulated results of repointing the Tetra-Star configuration worked exactly as the closed form solutions predicted, both in precessed angle, fuel consumed, and verifying the smoothness of the maneuver. The repointing maneuver requires the use of a variable thrust, sub-Newton propulsion system with a magnitude of 0.1 Newton. The sinusoidal thrust profile can be approximated step-wise if the resolution of the thrust steps is of the order of 0.001 Newton. A trade to consider, is of precessing the tethered constellation in a rougher, pulse-like manner and have the control system expend fuel to dampen the out-of-plane disturbances after the maneuver is completed. The simulations show, however, that a smooth re-point maneuver is possible and stable, driven in a completely open-loop fashion with variable thrust propulsion.

\section{Future studies}

Improvements in the modeling will include the use of bodies, not just point masses, and the modeling of tethers having mass with sufficient grid points to capture the first 2 transverse vibration modes. This will allow subtle out-of-plane motions to be studied. Also the inclusion of a control system with sensors and actuators to determine if 1 arc second of stability between subapertures is possible. Sensitivity of the parameters will be investigated, and the effect of transverse tether vibrations will be noted. If the transverse vibrations are problematical, then devices to dampen them actively will be sought.

Future configurations will focus on rigidly-shaped triangular arrangements, as the pendulum style configuration is overly sensitive to in-plane motions, though it is not ruled out by any means.

On the practical side, the tether material design will require investigation for maximizing the life of the tether, to account for environmental deterioration and micrometeoroid damage. Preliminary layouts and designs should be attempted for the winch mechanism, terminal damping shock strut, and the sub-aperture spacecraft itself for a more realistic mass estimate. A full evaluation of the system must include all of the closed-loop control systems which affect spacecraft attitude, mirror pointing, and wave-front control, as well as interior and exterior perturbation sources. The deployment sequence for a tethered observatory should be investigated. For example, a possible deployment scenario could include building, launching, and assembling of components in low earth orbit (LEO), then final launching to the L2 Lagrange point. When the stowed assembly is in L2 orbit, then the deployment and initialization of the tethered constellation can proceed. Perhaps the International Space Station will discover that its forte will be the assembly of large spacecraft, whose large components were built on the ground and boosted to LEO in the usual fashion. The mission design must be carefully conceived and scrutinized, as risk will be a palpable factor (the technique being so new), and must be an equal partner with all other parameters when mission trade studies are exercised. A technology validation mission will be needed.

\section{Last Words}

Spinning tethered constellations combined with synthetic aperture techniques can make impossibly large space-platform observatories come true. But nothing comes for free, as it will still have some complexities regarding optics, controls, and deployments to work out. Compare that to the rigid, massive alternatives. Surely this is an enabling technology that will revolutionize space-platform observation. 